\title{
A Review on Importance of DWDM Technology in Optical Networking
}

\author{
Anjana M.D ${ }^{1}$, Dr. Usha Rani K. $\mathrm{R}^{2}$ and Akshata Aski ${ }^{3}$ \\ ${ }^{1,2}$ RV College of Engineering, Bengaluru, India \\ ${ }^{3}$ Tejas Networks Limited, Bengaluru, India \\ ${ }^{1}$ anjanamahaveerd.ec17@rvce.edu.in ,2usharani@rvce.edu.in , \\ and ${ }^{3}$ akshathaa@tejasnetworks.com
}

\begin{abstract}
Optical communication uses light signals for transmitting information from source to destination. Optical fiber communication has been more popular for long-distance data transfer in recent years due to various benefits such as low loss, low cost, simple amplification, minimal interference, and light weight. A simple optical telecommunication system consists of a transmitter, a medium, and a receiver. Wavelength Division Multiplexing (WDM) is a significant improvement in optical communication. WDM is basically used for improving the spectral efficiency and to handle more data from several clients. Normal WDM, CWDM and DWDM are three types of WDM technology. DWDM uses most denser optical channels in optical networking. Complete detail about all types of WDM is given. Optical spectrum used in transmission of light signals is also discussed.
\end{abstract}

Keywords: optical communication, WDM, CWDM, DWDM, Mux, Demux, ROADM, PON.

\section{INTRODUCTION}

Because of several advantages such as low loss, cheap cost, easy amplification, low interference, and light weight, optical fiber communication has been increasingly popular for long-distance data transport in recent years. A transmitter, a medium, and a receiver make up a basic optical telecommunication system. The signal is converted into light waves by the transmitter. The light waves are then carried to their destination through a medium (optical fiber). The receiver at the destination converts the sent signal back to its original form. Dense Wavelength Division Multiplexing (DWDM) is a significant advancement in optical communication. Many optical devices used in DWDM networks (Reconfigurable Optical Add/Drop Multiplexer (ROADM), Mux, and so on) aggregate the output from several optical transmitters and transmit the resultant signal on a single optical fiber. Another DWDM device (Demux) splits the combined optical signals at the receiving end and sends each channel to an optical receiver. Between DWDM devices, just one optical fiber is used (per transmission direction). With DWDM, many optical channels can share a single fiber optic connection. A wavelength is assigned to each optical channel.

An optical add filter combines multiple wavelengths generated by distinct optical transmitters operating on various fibers into a composite signal that is sent on a single channel (Mux filter). An optical drop filter (Demux) splits all of the different wavelengths of the composite signal out to individual fibers at the receiving end. Mux and Demux (transmit and receive) components are usually housed in a single enclosure known as a Multiplexing and Demultiplexing Unit (MDU). 


\section{Optical Spectrum}

\subsection{Optical wavelength bands}

For long-distance communication, fiber optical communication is carried out in the infrared wavelength range. The wavelength range is from $1260 \mathrm{~nm}$ to $1675 \mathrm{~nm}$. In this range, optical signals have less loss and a higher data transmission capacity. Optical transmitters and receivers are designed to operate in this wavelength range. At longer wavelengths, optical signals exhibit less attenuation. However, wavelengths greater than $1675 \mathrm{~nm}$ do not prove to be efficient for fiber optic communication because infrared wavelengths transition between light and heat. As a result, surface temperature acts as background noise and disrupts signals at longer wavelengths.

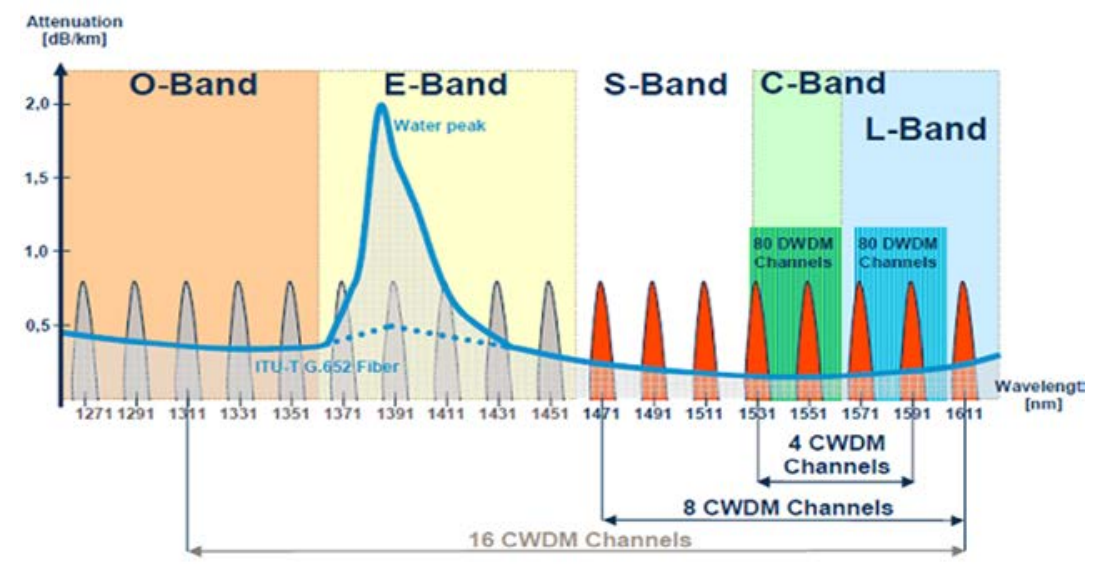

Figure 1. Optical spectrum

Table 1. Optical wavelength bands

\begin{tabular}{|c|l|c|}
\hline Band & Description & Wavelength range (nm) \\
\hline O-band & Original band & $1260-1360$ \\
\hline E-band & Extended band & $1360-1460$ \\
\hline S-band & Short wavelength band & $1460-1530$ \\
\hline C-band & Conventional band & $1530-1565$ \\
\hline L-band & Long wavelength band & $1565-1625$ \\
\hline U-band & Ultra-wavelength band & $1625-1675$ \\
\hline
\end{tabular}

After extensive research and testing, light with a wavelength of 1260-1625 nm has the lowest dispersion signal distortion with the lowest loss, making it ideal for transmission in optical fiber. The O-band was the first of these five wavelength bands to be used for fiber optic communication. In this band, signal dispersion due to chromatic dispersion is minimal. The loss of optical fiber in S-band is lower than in O-band, and it is used as the downstream wavelength in Passive Optical Network (PON). The E-band is the least used of the five wavelength bands. Because of residual water ( $\mathrm{OH}$ group) impurity in the glass (solid blue line in Figure 1), optical fiber exhibits the greatest attenuation in the E-band. The loss of commonly used optic fiber (International Telecommunications Union (ITU)-T G.652.D) in E-band has become less than that of O-band after using dehydration technique during glass production (dotted blue line in Figure 1). Nonetheless, E-band usage is limited because many optical networks were deployed prior to 2000.

Today, optical fiber has the lowest loss in the C-band and is suitable for long-distance transmission systems. It is commonly used in many metropolitan areas, Local Area 
Network (LAN), and other applications in conjunction with WDM, long-distance, ultralong-distance, and submarine optical transmission systems. L-band has the secondlowest loss and can be used when C-band is insufficient to meet the required bandwidth demand.

\subsection{WDM}

\section{Wavelength Division Multiplexing}

WDM is a technique used to increase the data transmission capacity of optical fiber. It is a technique for multiplexing various data streams, i.e., optical channels of varying wavelengths in terms of laser light colors, onto a single strand of fiber. WDM is carried out in the Infra-red (IR) region. Wavelength and frequency are merely synonyms for the same thing. The velocity of the carrier wave is defined as frequency (in $\mathrm{Hz}$ ) multiplied by wavelength (the physical length of one cycle). Because wavelength and frequency have an inverse relationship (shorter wavelength equals higher frequency), WDM and FDM use the same technology. As a result, WDM of optical signals of varying wavelengths is analogous to FDM of electric signals of varying frequencies. At the receiving end, a demultiplexer separates multiplexed signals into their original channels. A demultiplexer is frequently used as a filter to select a specific wavelength of an optical signal.

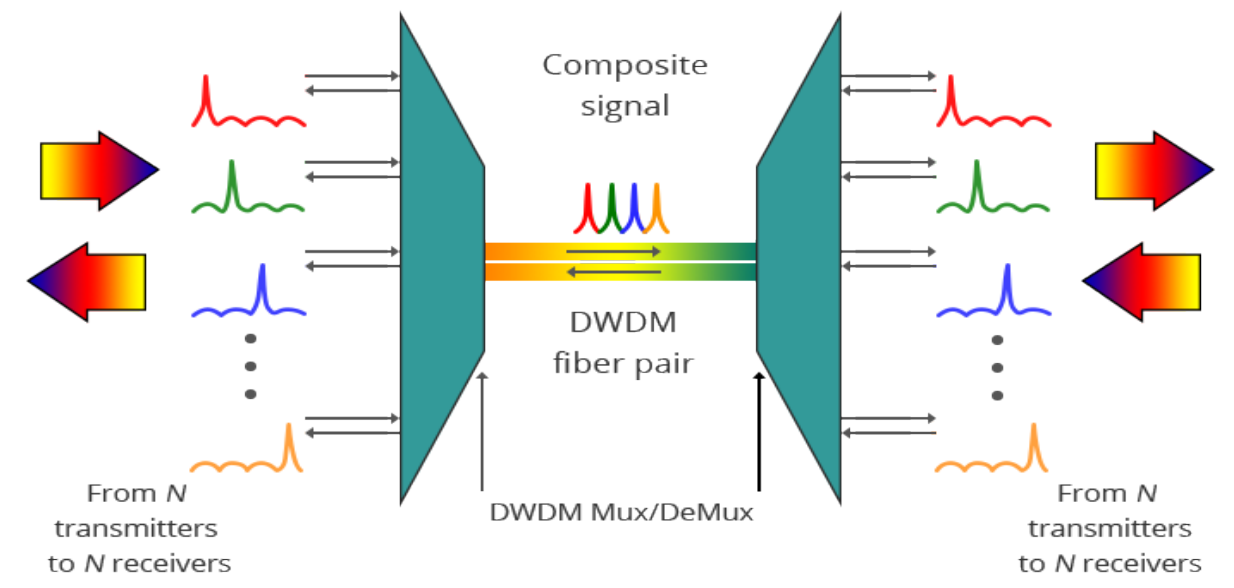

Figure 2. Multiplexing and demultiplexing of optical signals

WDM systems are currently widely used in telecommunications companies as a technology enables them, without any additional number of optical fibers, to expand network capacity. This WDM technique was first published in 1978 and WDM systems were produced in the laboratory by 1980 . There were only two signals in the first WDM system. Modern systems can multiply 160 signals, allowing a $100 \mathrm{Gbit} / \mathrm{s}$ basic system to be expanded to more than $16 \mathrm{Tbit} / \mathrm{s}$ over single fiber. A 320-channel system handling has also been implemented (12.5 GHz channel spacing).

\subsection{Types of Wavelength Division Multiplexing Technique}

WDM is classified into three types:

1) Normal WDM

2) Coarse Wavelength Division Multiplexing (CWDM)

3) Dense Wavelength Division Multiplexing (DWDM)

Normal WDM uses both $1310 \mathrm{~nm}$ and $1550 \mathrm{~nm}$ of normal wavelength. In optical wavelength spectrum, CWDM operates with less channel numbers $(4,8,16$, or 18$)$. When compared to DWDM, CWDM uses more channel spacing. In CWDM, spacing between the channels is $20 \mathrm{~nm}$. Also known as wide WDM is CWDM. CWDM is often used in 
applications with short distances. CWDM costs are less than the DWDM channel spacing. The spacing between two adjacent, optical channels is defined as the nominal frequency or wavelength variation. Inter-channel cross-speech is the minimum channel spacing and is connected to many factors: the channel bit rate, the modulation format, the filter pass band and changes to the center wavelength (due to laser manufacturing and laser temperature variations).

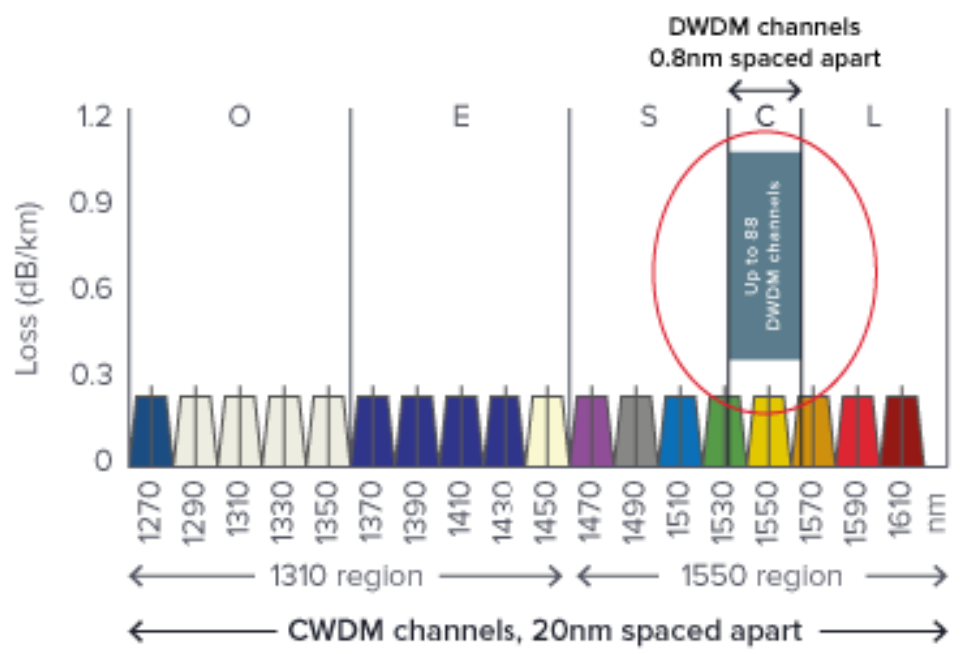

Figure 3. 18 CWDM channels

The channels of 18 CWDM with a distance of $20 \mathrm{~nm}$ in the range of 1260-1625 nm are displayed in Figure 3. Less channel spacing is used by DWDM compared to CWDM. In the DWDM technique, channel distances are specified as a frequency. The spacing of the DWDM channel is $0.8 / 0.5 \mathrm{~nm}$. It's small channel distance enables more information to be transmitted simultaneously. In the C-band, $100 \mathrm{GHz}$ spacing of the channel allows up to 40 channels. It is possible to use $320 \mathrm{C}$-band channels with $12.5 \mathrm{GHz}$ channel spacing. Also known as Ultra-DWDM. Figure 4 shows few DWDM channels with $100 \mathrm{GHz}$ channel spacing in C-band (both frequency and wavelengths).

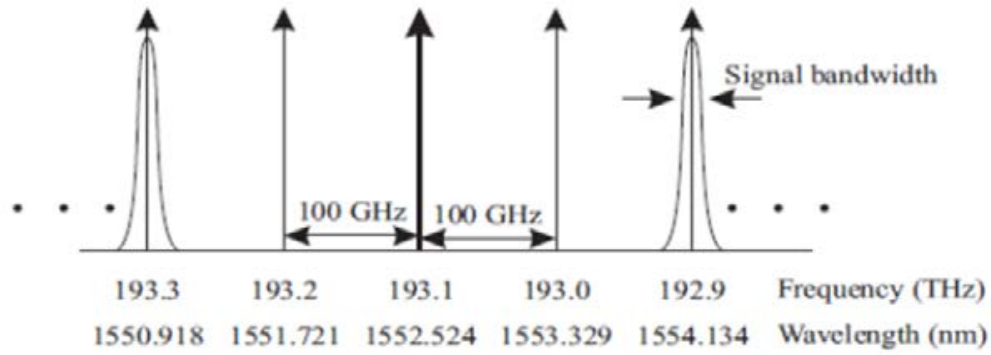

Figure 4. DWDM channels with $100 \mathrm{GHz}$ spacing

\section{DWDM Network}

\subsection{DWDM Network Architecture}

In optical fiber communication DWDM is an important extension. DWDM (Mux) multiplex the output for transmission over a single optical fiber by multiple optical transmitters. On the receiving end, the multiplexed optical signals and each optical 
signal is transmitted to an optical receiver via another DWDM device (demux). Between DWDM devices only one optical fiber is used (per transmission direction).

Figure 5 illustrates a typical DWDM network. Any type of traffic such as SDH, SONET and Ethernet could be transmitted by DWDM network. The traffic is converted into optical form by means of optical transmitter and transponders other than optical signal, DWDM which matches wavelengths. DWDM systems can transmit voice transmission, e-mail, video and multimedia data. DWDM network basic components are:

1) Optical transmitters/receivers

2) Multiplexers/Demultiplexers

3) Reconfigurable Optical Add/Drop Multiplexers (ROADM)

4) Optical amplifiers

5) Optical Power Monitor (OPM)

6) Inter-Leaver Unit (ILU)

7) Optical Transponders

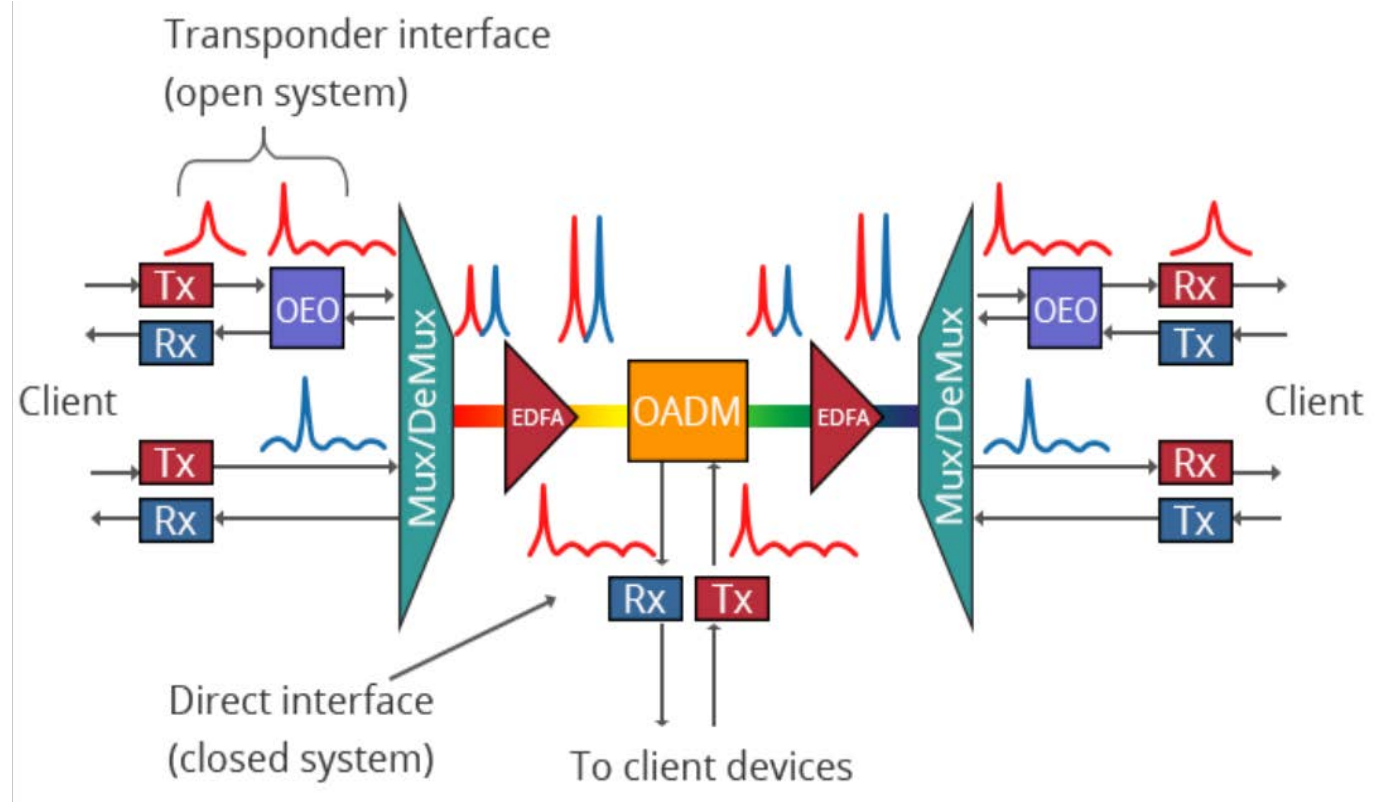

Figure 5. Typical DWDM network

4.2.1 Optical transmitters/receivers: Optical transmitter is located on transmission end of fiber cable and optical receiver is located on the other end. Transceiver (SFP, $\mathrm{SFP}+$ ) is a device which works as both transmitter and receiver. The optical transmitter converts an electric signal to an optical signal using an LED or laser diode. The original signal's content remains unchanged. Total internal reflection allows light waves or optical signals to travel across the optic fiber. As an optical receiver, a photodetector is used. It converts optical signals to electrical signals.

4.2.2 Multiplexers/Demultiplexers: An optical add filter combines multiple wavelengths generated by different optical transmitters operating on different fibers into a single composite signal and transmits it on a single channel (Multiplexer). An optical drop filter (Demultiplexer) separates all of the individual wavelengths of the composite signal out to individual fibers at the receiving end.

4.2.3 Reconfigurable Optical Add/Drop Multiplexers (ROADM): ROADM works in the same way as optical add/drop MDU. Only difference is that it can 
handle optical signals containing 2 or more wavelengths at add/drop ports. This device also works on WDM technology. It is used for remotely switching traffic from WDM system at the wavelength layer. WSS is used for remote switching of WDM traffic. ROADM provides reconfigurability to the network.

4.2.4 Optical Amplifiers: Optical amplifiers either amplify or boost a signal, or they add gain to optical signals. These devices work by supplying additional energy to photons in a light signal. Light amplification occurs without converting the optical signal to an electrical signal and is an extremely important device that supports today's long-distance optical communication networks. Amplifiers can operate on a variety of wavelengths. As a result, these devices can be used with any other DWDM components. Amplifiers are one-way devices.

4.2.5 Optical Power Monitor (OPM): It is a device used for testing average power in fiber optic systems. OPM monitors power levels of the optical signal at any port on a device. It can be used with other DWDM components like transmitters/receivers, mux/demux, amplifiers etc. In MDU, monitoring port is used for this purpose. When the strength of the optical signals is less than the required level, then alarms will be reported.

4.2.6 Inter-Leaver Unit (ILU): The ILU is a three-port passive optical device that is used to interleave odd and even DWDM channels. In general, it is utilized to create denser optical channels. Odd and even DWDM channels with $100 \mathrm{GHz}$ spacing, for example, can be interleaved to produce DWDM channels with $50 \mathrm{GHz}$ spacing. Similarly, more dense optical channels with spacing of $25 \mathrm{GHz}$ or 12.5 $\mathrm{GHz}$ can be built. De-Inter leaver unit works exactly opposite to ILU.

\section{Conclusion}

High data rate in long distance communication is basic requirement in telecommunication networks. WDM achieves this requirement using less resources. WDM technology and types of WDM are explained in detail in this paper. DWDM network architecture is shown and functionality of basic components used in DWDM network are also discussed.

\section{REFERENCES}

[1] Joseph M. Kahn, Keang-Po Ho, "Spectral Efficiency Limits and Modulation/Detection Techniques for DWDM Systems", IEEE JOURNAL OF SELECTED TOPICS IN QUANTUM ELECTRONICS, VOL. 10, NO. 2 (2014).

[2] Pravesh Kumar Chaudhary, Ravi Kumar, Sumit Kaushik, "Review Paper on DWDM Technology”, International Journal of Engineering Science and Computing, volume 8, issue 12 (2018).

[3] T. Kabilan, N. Victor jaya, "Performance Analysis of 48 Channels DWDM System using EDFA for Long Distance Communication”, - Global Research and Development Journal for Engineering, volume 2, issue 3 (2017).

[4] Diksha Sharma, Ashish Sachdeva, "Wavelength Division Multiplexing”, International Journal of Advanced Research in Electronics and Communication Engineering (IJARECE) Volume 5, Issue 5 (2016).

[5] Vivek Kachhatiya, Shanthi Prince, "Wavelength Division Multiplexing-Dense Wavelength Division Multiplexed Passive Optical Network (WDM-DWDM-PON) for Long Reach Terrain Connectivity", International Conference on Communication and Signal Processing (2016). 
[6] Samael Sarmiento, Jose A. Altabas, David Izquierdo, Ignacio Garces, Salvatore Spadaro, and Jose A. Lazaro, " Cost-Effective DWDM ROADM Design for Flexible Sustainable Optical Metro-Access Networks" J. OPT. COMMUN. NETW./VOL. 9 (2017).

[7] S. Papaioannou D. Fitsios G. Dabos, " On-Chip Dual-Stream DWDM EightChannel-Capable SOI-Based MUXs/DEMUXs With 40-GHz Channel Bandwidth", IEEE photonics journal (2015).

[8] Jiannan Zhu, Stephan Pachnicke, Mirko Lawin, Stephen Mayne, Adrian Wonfor, Richard V. Penty, Rosie Cush, Richard Turner, Paul Firth, Mike Wale, Ian H. White, and Jörg-Peter Elbers, "First Demonstration of a WDM-PON System Using Full Cband Tunable SFP+ Transceiver Modules”, J. OPT. COMMUN. NETW./VOL. 7 (2015).

[9] Sandis Spolitis, Inna Kurbatska, Vjaceslavs Bobrovs, "Comparison of C-band and Lband WDM-PON systems performance with PAM-4 modulation format", International Workshop on Fiber Optics in Access Network (FOAN) (2017).

[10] A. Dochhan, H. Griesser, M. H. Eiselt, J.-P. Elbers, "Solutions for $80 \mathrm{~km}$ DWDM Systems”, JOURNAL OF LIGHTWAVE TECHNOLOGIES (2015). 